\title{
Modelo de gestión técnico ambiental para la formalización de la minería a pequeña escala - Caravelí - Arequipa
}

\author{
Environmental technical management model for the formalization of small \\ mining - Caravelí - Arequipa
}

Carlos Santos Alvarado Quispe ${ }^{1}$

Recibido: 15/02/2021 - Aprobado: 01/09/2021 - Publicado: 23/12/2021

\begin{abstract}
RESUMEN
Se analiza la problemática de la formalización de la minería en pequeña escala: pequeña minería y minería artesanal, especialmente a la aplicación de la gestión técnico - ambiental, en Caravelí-Arequipa, identificándose que el problema reside en el uso y lentitud, alcanzándose una propuesta de mejora de los procesos de formalización de la actividad minera, realizando comprobación del proceso y su efectividad, apreciando la problemática y proponiendo una mejora en lo normativo y operativo, que considera incentivos, mejoras, tecnificación y gestión ambiental eficiente, para ello se ha analizado la situación de la minería en Caravelí - Arequipa a fin de optimizar la gestión en la formalización de sus actividades mineras. Se pudo apreciar que esta formalización tiene años de realizarse y no tiene avances favorables, por lo que el análisis fue necesario y permitió identificar los principales problemas proponiendo una gestión más efectiva y eficiente, cumpliéndose el propósito de la investigación, estos arreglos que son las mayores dificultades se refieren a: autorización de uso del terreno superficial y la titularidad, contrato de explotación y/o cesión sobre la concesión minera. La investigación ha cumplido con todo lo necesario para poder apreciar el estudio de forma clara para poder comprobar el resultado a obtener.
\end{abstract}

Palabras claves: Formalización minera; impactos ambientales; minería; minería artesanal; pequeña minería; problemática de la minería.

\begin{abstract}
The problem of the formalization of small-scale mining is analyzed: small mining and artisanal mining, especially to the application of technicalenvironmental management, in Caraveli - Arequipa, identifying that the problem lies in the use and slowness, reaching a proposal of improvement of the processes of formalization of the (mining) activity, checking the process and its effectiveness, appreciating the problem and proposing an improvement in the normative and operational, which considers incentives, improvements, technification and efficient environmental management, for this the situation of the mining in Caraveli - Arequipa in order to optimize the management in the formalization of its (mining) activities. It was observed that this formalization has been carried out for years and has not had favorable advances, so the analysis was necessary and allowed to identify the main problems proposing a more effective and efficient management, fulfilling the purpose of the investigation, these arrangements that are the greatest Difficulties refers to: authorization of use of the surface land and ownership, exploitation contract y/o cession on the mining concession. The research has fulfilled everything necessary to be able to appreciate the study clearly to verify the result to be obtained.
\end{abstract}

Keywords: Artisanal mining; environmental impacts; mining; mining formalization; mining problems; small mining. 


\section{INTRODUCCIÓN}

Las actividades mineras informales de pequeña escala, como la pequeña minería y minería artesanal, que no debe confundirse con minería ilegal, sobre la actividad de la pequeña minería se realizó el análisis de esta actividad informal que es producto de la falta de trabajo y de la necesidad manifiesta en las poblaciones vulnerables, que se dedican a este tipo de actividad, al respecto Luján (2008), precisa: "La minera artesanal es una actividad generadora de empleo y un medio de vida para nosotros los mineros artesanales; y eso nos convierte en trabajadores que debemos asumir responsabilidades junto con nuestras familias y con nuestras comunidades, debiendo trabajar con compromiso para que nuestra actividad sea sostenible en el tiempo y no destruya la naturaleza" (Luján, 2008). Las actividades mineras informales ocasionan serios impactos ambientales, debido a la falta de con ambiental, al desconocimiento o desinterés de aplicar tecnologías apropiadas o limpias, "Actualmente tenemos muchas deficiencias en el desarrollo de nuestras labores; por eso necesitamos capacitarnos en el uso de tecnologías limpias que no contaminen nuestro ambiente y que nos ayuden a recuperar mayor cantidad ;La vida es más preciosa que el oro! de metal, pues estamos perdiendo mucho oro por no tener métodos mejores (Luján, 2008)”.

Esta investigación tiene como propósito ser el referente como modelo teórico de la explicación de hechos y fenómenos que se generalicen a partir del problema de investigación y un aporte en favor de este procedimiento técnico - ambiental tan necesario para la gestión de la pequeña minería y minería artesanal.

Al respecto de la gestión técnico ambiental, se tiene la siguiente referencia de la Institución (TECSUP, 2020):

"Son competencias profesionales que permiten el empleo de diversos recursos tecnológicos para la planificación, el diseño y la ejecución de las operaciones unitarias mineras, ya sea en labores a cielo abierto o en labores subterráneas, logrando con ello un desempeño laboral exitoso en el complejo entorno productivo de la industria minera peruana". (TECSUP 2020).

El Ministerio Del Ambiente (2017), establece que la Gestión Ambiental es:

\begin{abstract}
“... es un proceso permanente y continuo, constituido por el conjunto estructurado de principios, normas técnicas y actividades, orientado a administrar los intereses, expectativas y recursos relacionados con la actividad minera y los objetivos de la política nacional del ambiente, para alcanzar una mejor calidad de vida y el desarrollo integral de la población, el desarrollo de las actividades económicas y la conservación del patrimonio ambiental y natural del país (MINAM, 2017).
\end{abstract}

La minería formal actual contribuye al desarrollo del país de forma integral, puesto que genera ingresos al país por impuestos y por acciones de responsabilidad social, además de ser regulada actualmente para realizar actividades, pero sin afectar al ambiente. De acuerdo a lo que precisa (Díaz Lazo, 2019) sobre la sostenibilidad en el desarrollo minero: "Es imprescindible el desarrollo de un marco de indicadores ambientales más amplio y especifico para tres (03) estratos de la minería (la gran minería, mediana minería y pequeña minería), de tal manera que se sumen esfuerzos para alcanzar la sostenibilidad en la industria minera peruana” (Díaz Lazo, 2019, pág. 43)

Es preciso anotar como se considera el rol de la Pequeña minería y Minería artesanal frente al problema de formalización y ambiental, como lo señala Romero y otros (2016), en el que precisan como la minería artesanal, principalmente, ha causado un problema de contaminación y es el Estado en su afán de resolver esa situación ha emitido normas como la Ley $\mathrm{N}^{\circ}$ 27651, Ley de Formalización y Promoción de la Pequeña Minería y Minería Artesanal y la Ley $\mathrm{N}^{\circ}$ 27446, Ley del Sistema Nacional de Impacto Ambiental, y los relaciona con la elaboración de una Declaración de Impacto Ambiental y Estudios de Impacto Ambiental (Romero A., 2014, pág. 93), no cabe duda que es necesaria la investigación para determinar los avances y mejorar el proceso en sí como el artículo propone.

\section{Instrumentos de Gestión Ambiental}

Constituyen medios operativos que son diseñados, normados y aplicados con carácter funcional o complementario, para efectivizar el cumplimiento de la Política Nacional Ambiental y las normas ambientales que rigen en el país. Al respecto la OEFA precisa: "Mecanismo orientado a la ejecución de la política ambiental, sobre la base de los principios establecidos en la Ley General del Ambiente y sus normas complementarias y reglamentarias. Estos instrumentos pueden ser de planificación, promoción, prevención, control, corrección, información, financiamiento, participación, fiscalización, entre otros" (OEFA, 2016).

\section{Procesos de gestión de la formalización}

Los procesos de Formalización Minera Integral están incluidos en el D.L. $\mathrm{N}^{\circ} 1336$, que indica:

a. Definición de la minería formal e informal. Identificando su accionar y limitaciones en ambos casos con respecto a la explotación minera.

b. Identificación de la categoría de intervención, siendo ellos: Titular minero, Comercializador y Plantas de beneficio.

c. Instrumentos de gestión ambiental para la Formación de Actividades de Pequeña minería y Minería artesanal (IGAFOM), que tienen dos aspectos:

- Correctivo: Formato de Declaración Jurada y son medidas que se mencionan ante el Registro Integral de Formalización Minera.

- Preventivo: Que se declaran igualmente al inscribirse al Registro Integral de Formalización Minera. 
d. Expediente técnico, a partir de la promulgación del D.S. N ${ }^{\circ}$ 018-2017-EM establece y describe su contenido y la simplificación de los hechos en beneficio del trámite.

e. Autorización de Inicio o Reinicio de Actividades mineras de explotación y/o Beneficio de Minerales y/o Título de Concesión de Beneficio, basado en el D.S. $N^{\circ} 018-2017-E M$ y el D.L. N $^{\circ}$ 1336 y complementarias.

f. Requisitos y pasos para la Formalización de la Actividad Minera de la Pequeña Minería y Minería Artesanal:

- Acreditación de Propiedad o autorización de uso del terreno superficial, de acuerdo con el Título III del presente Decreto Supremo.

- Acreditación de Titularidad, contrato de cesión o contrato de explotación respecto de la concesión minera, de acuerdo con el Título IV del presente Decreto Supremo.

- Presentación de Declaración jurada de inexistencia de restos arqueológicos, de acuerdo con el párrafo 3.2 del artículo 3 del Decreto Legislativo $\mathrm{N}^{\circ} 1336$.

- Aprobación del Instrumento de Gestión Ambiental para la Formalización de Actividades de Pequeña Minería y Minería Artesanal - IGAFOM o del Instrumento de Gestión Ambiental Correctivo - IGAC, de acuerdo con la normativa complementaria especial que sobre la materia se expide, mediante Decreto Supremo.

- Presentación del Expediente Técnico. (D.S. $\mathrm{N}^{\circ}$ 018-2017-EM).

La problemática por la que es importante considerar este aspecto se aprecia en las evidencias de:

- Contaminación por el uso inadecuado del Mercurio,

- Acumulación de cascajo y grava que conlleva la eliminación de la cobertura vegetal,

- $\quad$ La deforestación y

- La contaminación de los cursos de agua por sólidos en suspensión y la turbidez de estos.

Siendo en los yacimientos primarios (vetas), debido a:

- La acumulación de desmonteras inestables y generación de aguas ácidas, y

- La utilización sin criterio técnico de reactivos peligrosos como: mercurio y cianuro, entre otros. Como indican (Loaiza et al., 2008).

"Aunque se teme al cianuro como una sustancia mortal, en realidad es una sustancia química ampliamente utilizada y esencial para el mundo moderno. La clave para su uso seguro es la implementación de prácticas seguras de manejo" (Loaiza et al., 2008).

El estudio ha de apreciar que existen dificultades como:

A. Falta de personal especializado y logística, en las instancias del Estado.

B. Inadecuada planificación del Proceso de Formalización de estas actividades mineras.

Cabe mencionar que el nuevo proceso de formalización minera integral que se ha implementado tiene el objetivo de insertar al estado de derecho a un gran volumen y potencial de mineros, que han encontrado en esta actividad su fuente de trabajo, teniendo que el Estado debe hilar muy fino para evitar los conflictos y pueda reinar la paz social para todos los actores involucrados, pues los conflictos son los que principalmente afectan los hechos de manera imprevista, por lo que es importante señalarlo, de acuerdo a lo que Arbaiza señala: “...por lo que es necesario ejecutar las actividades mineras respetando el medio ambiente, con seguridad e higiene para las personas y cuidado de la salud de los trabajadores, a fin de evitar posibles conflictos sociales y al final de la vida útil recuperar el paisaje natural. De este modo se garantiza el desarrollo sostenible de la actividad. La propuesta de la investigación es desarrollar un sistema de Gestión Técnica y Ambiental, la cual consistirá en determinar qué instrumentos técnicos $y$ ambientales deben considerar los mineros informales en materia de protección ambiental, para asegurar que en el desarrollo de sus actividades se tenga en cuenta la prevención y la minimización de los efectos sobre el entorno" (Meza, 2014).

Es necesario comprender que la pequeña minería y la minería artesanal carece de tecnología de avanzada en exploración y explotación, que se hace una situación tan necesaria y urgente, como lo establece (Torres Guerra et al., 2021): "Es un reto importante que la minería en nuestro país se inserte de forma integra en la automatización $y$ digitalización en todos los procesos de la industria minera, a través de la geometalurgia, que abarca desde la exploración hasta la operación. De tal manera, que estas innovaciones incidan significativamente en la productividad, seguridad y la capacidad de procesamiento de la información, para resolución de problemas con decisiones más acertadas, que brinden valor económico al negocio minero" (Torres Guerra et al., 2021).

\section{MÉTODOS}

La investigación es del tipo descriptiva correlacional, “... que consiste en la caracterización de un hecho, fenómeno, individuo o grupo, con el fin de establecer su estructura o comportamiento, este tipo de investigación es de nivel intermedio por la profundidad de los conocimientos y es explicativa" (Arias Odón, 2010), que presenta el siguiente diseño: 
Correlacional.

$M \quad \begin{aligned} & \mathrm{O}_{x} \\ & \mathrm{O}_{y}\end{aligned}$

Dónde:

$\mathrm{M}=$ Muestra, que son los mineros informales en vías de formalización.

$\mathrm{O}_{\mathrm{x}}=$ Observación de los procesos de formalización minera.

$\mathrm{O}_{\mathrm{y}}=$ Observación de los mineros formalizados.

$\mathrm{r}=$ Relación entre ambas variables.

Se determinó que la gestión técnica intervendría en la formalización de la pequeña minería y minería artesanal con el número de mineros formalizados que cuenten con la autorización de inicio/reinicio de operaciones mineras y la gestión ambiental contribuiría con la formalización de la pequeña minería y minería artesanal alcanzando la conservación del patrimonio ambiental y natural del país, y una mejor calidad de vida de la población, minimizando la contaminación minera en aire, agua y suelo, como parte de ese proceso se considera que la Gerencia Regional de Energía y Minas de Arequipa, debe asumir el reto de manera responsable para contribuir eficientemente con esta nueva orientación y estrategia, haciendo un trabajo de monitoreo permanente y coordinado del estado (Gobiernos local, regional y central) hacia los mineros informales, ya que ellos según la Ley 27651, son los que tienen las facultades y competencias de ejecutar el nuevo paquete de normativa minera para la minería a pequeña escala (Medina Cruz, 2014).

El estudio se realizó en 32 mineros informales de actividad minera de explotación subterránea de la provincia de Caravelí, región de Arequipa, realizada de manera aleatoria y circunstancial.

Se aplicaron técnicas como la:
A. Observación,
B. Análisis de documentos y Encuestas.
C. Empleando instrumentos como:
D. Registro del análisis documental y
E. Entrevista y Encuesta a los mineros.

La investigación se ha dividido en tres etapas:

1. Recopilación de la información existente: Consistió en buscar toda la información referente a la zona de estudio y a las actividades que debían desarrollarse en la misma. Esta etapa se desarrollará en diversas organizaciones de las regiones de estudio.

2. Trabajo de campo: En el que se realiza el levantamiento de información a través de encuestas y entrevistas a los mineros informales de la provincia minera de Caravelí, presentes en la región de Arequipa.

3. Trabajo de gabinete: Consistirá en las siguientes actividades:

a. Realización del acopio de información en la provincia de Caravelí, en la región de Arequipa, zona del estudio.

b. Aplicación de las entrevistas y encuestas, ajustado al modelo de entrevista del Ministerio de Energía y Minas, complementado con preguntas relevantes al presente estudio.

c. Elaboración de información georreferenciada en un Sistema de Información Geográfica (SIG) y se ha trabajado en el sistema de coordenadas Universal Transversal de Mercator (UTM) y Datum WGS 84 (Sistema Geodésico Mundial 1984), Zona 18 S, haciendo el mapeo de la zona en la que se desempeñan los mineros informales.

\section{RESULTADOS}

Entre los resultados tenemos la Tabla 1, Tabla 2 y Figura 1.

Tabla 1. Mineros informales inscritos a nivel nacional, según personería

\begin{tabular}{|c|c|c|c|c|}
\hline Región & Persona natural & $\%$ & Persona jurídica & $\%$ \\
\hline Amazonas & 166 & 0.28 & 48 & 0.42 \\
\hline Ancash & 2874 & 4.93 & 902 & 7.82 \\
\hline Apurímac & 6007 & 10.31 & 442 & 3.83 \\
\hline Arequipa & 12028 & 20.65 & 1949 & 16.91 \\
\hline Ayacucho & 6254 & 10.74 & 798 & 6.92 \\
\hline Cajamarca & 719 & 1.23 & 281 & 2.44 \\
\hline Callao & 13 & 0.02 & 10 & 0.09 \\
\hline Cusco & 2495 & 4.28 & 499 & 4.33 \\
\hline Huancavelica & 1114 & 1.91 & 326 & 2.83 \\
\hline Huánuco & 575 & 0.99 & 167 & 1.45 \\
\hline Ica & 1766 & 3.03 & 439 & 3.81 \\
\hline Junín & 848 & 1.46 & 401 & 3.48 \\
\hline La Libertad & 5177 & 8.89 & 812 & 7.04 \\
\hline Lambayeque & 282 & 0.48 & 67 & 0.58 \\
\hline Lima & 2036 & 3.50 & 831 & 7.21 \\
\hline Loreto & 70 & 0.12 & 30 & 0.26 \\
\hline Madre de Dios & 5700 & 9.79 & 895 & 7.76 \\
\hline Moquegua & 188 & 0.32 & 65 & 0.56 \\
\hline Pasco & 869 & 1.49 & 184 & 1.60 \\
\hline Piura & 1409 & 2.42 & 363 & 3.15 \\
\hline Puno & 7053 & 12.11 & 1822 & 15.80 \\
\hline San Martín & 155 & 0.27 & 41 & 0.36 \\
\hline Tacna & 306 & 0.53 & 111 & 0.96 \\
\hline Tumbes & 114 & 0.20 & 22 & 0.19 \\
\hline Ucayali & 34 & 0.06 & 23 & 0.20 \\
\hline Total & 58252 & 100.00 & 11528 & 100.00 \\
\hline
\end{tabular}

Fuente: PAD MINEM http://pad.minem.gob.pe/REINFO_WEB/Index.aspx 06/09/2020 
Comentario de tabla 1: Se puede apreciar que el REINFO tiene inscritos a junio del 2020 a 58252 mineros inscritos como persona natural, de ese valor 12028 que son el $20,65 \%$ a nivel nacional que corresponden a Arequipa y 11528 como persona jurídica, de ese valor 1949 que son el $16,91 \%$ a nivel nacional y corresponden a Arequipa.

Tabla 2. Mineros informales inscritos a nivel regional - Región Arequipa

\begin{tabular}{lcc}
\hline \multicolumn{1}{c}{ Provincias } & Inscritos REINFO & $\%$ \\
\hline Arequipa & 829 & 5.93 \\
Camaná & 2719 & 19.46 \\
Caravelí & 6270 & 44.87 \\
Castilla & 875 & 6.26 \\
Caylloma & 407 & 2.91 \\
Condesuyos & 2719 & 19.46 \\
Islay & 93 & 0.67 \\
La Unión & 63 & 0.45 \\
Total & 13975 & 100.00 \\
\hline
\end{tabular}

Fuente: PAD MINEM. http://pad.minem.gob.pe/REINFO_WEB/Index.aspx. 06/09/2020

Comentario de Tabla 2: Se puede apreciar que el REINFO tiene inscritos a junio en lo que respecta a la región Arequipa a 13975 personas, de ellas se tiene inscritos en el REINFO 6270 mineros que corresponden al 44.87\%.

Comentario de Figura 1: Se puede apreciar en el REINFO que en la región Arequipa 13975 mineros son informales, de ese grupo se aprecia que 6270 mineros son informales, de ellos el $44,87 \%$ son de la provincia de Caravelí del total de esa región, siendo la concentración más alta de mineros informales en esa región.
Se ha aplicado una entrevista a 8 expertos en el tema del Proceso de formalización, a los que se identificó de acuerdo con sus capacidades y/o habilidades como 6 con un nivel de Bueno en el tema y 2 con el nivel Muy bueno en el tema, de experiencia, preparación y conocimiento, además se ha accedido a las siguientes respuestas de los 8 expertos (ver Figura 1):

- La mayoría de los 8 expertos consideran adecuada o algo adecuada el D.L. $\mathrm{N}^{\circ} 1293$ y el D.L. $\mathrm{N}^{\circ}$ 1336, ambas direccionadas a considerar de interés nacional el proceso de formalización.

- Todos coinciden en que el Proceso más complicado es la Acreditación de la titularidad de la concesión minera.

- Todos coinciden en que la corrupción es un aspecto que significa un impedimento para la formalización, los expertos marcaron hasta dos opciones, la otra opción se distribuyó entre: procesos complejos, conflictos de intereses entre los estamentos del Estado y autoridades contemplativas, al respecto es necesario considerar los siguientes aspectos, que confirman sus respuestas por las observaciones manifestadas: Existe rotación de personal, Trámites complejos, Alta rotación de funcionarios, Enquistadas en las zonas de minería ilegal. Controlan la cadena productiva, Autoridades regionales sin capacidad técnica, Nivel nacional no hay coherencia entre oficinas, El Estado desconoce la verdadera problemática de la MA/PE y consideran erróneamente: que la pequeña minería es una fuente de riqueza, cuando solo es una minería de subsistencia, no es consciente que

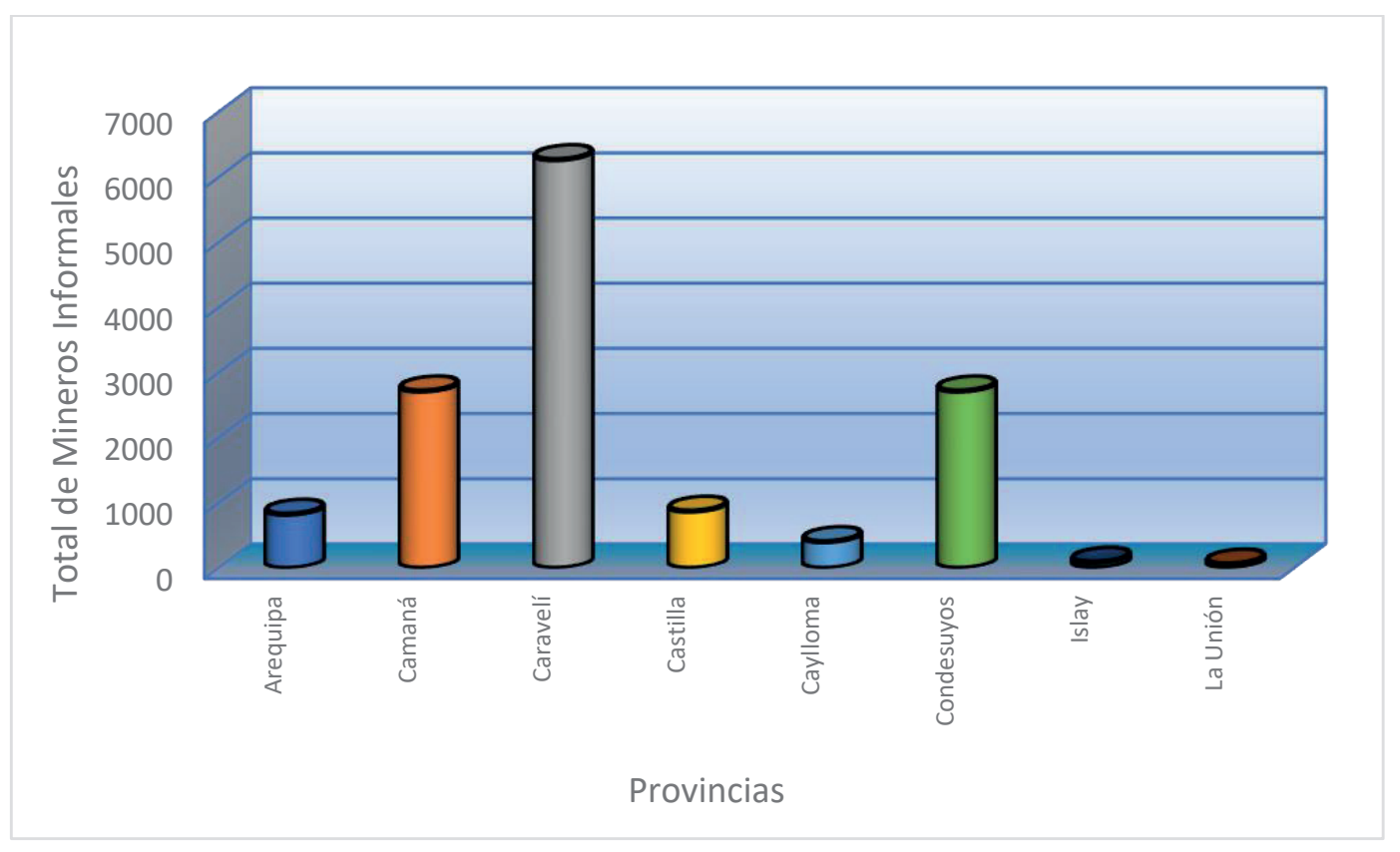

Figura 1. Distribución de Mineros informales por provincias en Arequipa.

Fuente: Elaboración propia 
son condiciones de trabajo muy adversas, no consideran que elaborar un plan de cierre ( $\sin$ el debido asesoramiento) es complicado de cumplir por un pequeño minero o minero artesanal, las interdicciones provocan daño al minero, el hecho de asumir tributaciones genera desconfianza porque siempre han sentido abuso de parte del Estado, otras falencias son la falta de personal técnico competente en el Estado, mucho manejo político y corrupción, y Direcciones Regionales de Energía y Minas, que son mesa de partes en muchos casos y la Dirección General de Formalización Minera ha burocratizado desde siempre los trámites y no solucionan rápidamente los pedidos de los mineros.

- La mayoría (5 de 8 expertos que equivale a $62.5 \%$ ), además coincide, que es necesario la capacitación para formalizar la pequeña y minería artesanal.

- No consideran que sea un incentivo el canje con impuestos en la fase inicial para la formalización, se debe buscar otro mecanismo.

A los mineros se les practicó una encuesta, de la que se tiene los siguientes resultados:

- Los mineros tienen experiencia diversa de los mineros encuestados es de $34 \%$ que se dedican hasta 5 años, $22 \%$ de 5 a 10 años, $13 \%$ de 10 a 19 años y $31 \%$ más de 20 años.

- $\quad$ El $72 \%$ de los mineros encuestados manifiestan haberse inscrito el año $2017,22 \%$ se inscribieron el año 2018 y el 6\% que lo hicieron el año 2019.

- Ante la consulta de "Si es una fuente de riqueza" el $6 \%$ de los mineros encuestados consideran que es una fuente de riqueza, para el $3 \%$ no significa riqueza absolutamente, un $72 \%$ precisa que No, que solo es para subsistir, 19\% prefiere no contestar.

- Ante la consulta si las normas ayudan a la formalización responden, el 3\% de los mineros encuestados consideran que, Si ayuda a la formalización, un $72 \%$ precisa que $\mathrm{Si}$, pero no mejoraría la situación, $25 \%$ prefirió no contestar.

- Con respecto al trato que le ofrecieron en el Proceso de formalización, el 38\% de los mineros encuestados respondieron que fue bueno, $56 \%$ indica que no fue bueno y $6 \%$ prefirió no contestar.

- Los mineros indicaron que la Dificultad para cumplir con la formalización: para el $6 \%$ considera que el D.L. 193 no es objetivo, 9\% que el formato IGAFOM no es claro en su aplicación, $\mathbf{4 1 \%}$ indican que es la acreditación de la titularidad de la concesión minera, el $16 \%$ indican que el Plan de Manejo Ambiental no debe ser requisito, el $28 \%$ considera que es la Acreditación del uso del terreno superficial, otras respuestas tienen el $0 \%$.

- $\quad$ Por otro lado, el $72 \%$ de los mineros consideran que el proceso de formalización es muy complejo, $19 \%$ consideran que es algo apropiado y solo un 9\% lo consideran apropiado.

- Los mineros encuestados referente a lo que consideran el mayor impedimento, el 59\% indican que es la corrupción, el 16\% que son los Procesos administrativos muy complejos, el 13\% que son las Autoridades, un $3 \%$ que existe un Conflicto de intereses y un $9 \%$ prefiere no opinar.

- Los mineros encuestados referente a la capacitación o Sensibilización para apoyar la Formalización tiene como respuestas: 75\% consideran que es necesario, 9\% indican como muy necesario, y otro $9 \%$ que es algo necesario, pero no urgente, $3 \%$ lo consideran Necesario y otro 3\% Prefiere no opinar.

\section{DISCUSIÓN}

De estudios realizados que tienen cierta similitud con el realizado se tiene las siguientes apreciaciones:

A. (Díaz Tobar, 2013) en su tesis presenta como una de las conclusiones: "Según lo investigado, existe concordancia en que las principales dificultades del sector tienen que ver principalmente, con la dificultad de constituir propiedad minera, el desconocimiento de sus reservas y las dificultades para la explotación de su faena (escasa mecanización e infraestructura, bajos estándares de seguridad, nulo acceso a crédito, etc.) y un bajo nivel de capital humano empresarial y laboral, dadas las características propias del perfil del Minero (baja escolaridad, nivel socioeconómico, exclusión social, etc.)" (Díaz Tobar, 2013). (Diseño de una estrategia de intervención basada en el desarrollo de capital humano para la minería artesanal Apreciación: En el estudio se hace referencia de que los problemas principales son la Titularidad y el Uso de la Superficie, entre otras que Díaz menciona, puesto que otros hechos son obvios, pero para el Informal minero peruano la mayor dificultad es la Acreditación de la titularidad de la concesión minera y la Autorización para hacer el uso de la superficie.

B. Guzmán et al. (2019) en su tesis presenta como una de sus conclusiones. "Los mineros artesanales e informales desconocen a fondo la legislación colombiana en relación con la minería y su trayecto histórico; donde se han vulnerado derechos fundamentales entorno al trabajo, a la vida, la propiedad, entre otros, su transición a la legalidad es lenta y compleja como se demostró, ante las inexactitudes y constantes cambios que se han venido presentando" (Guzmán et al., 2019). 
Apreciación: Con respecto a este caso, al margen del desconocimiento, los mineros advierten como problemas críticos la corrupción en el estudio también se este hecho tiene que ver con la vulneración del derecho, y algunos establecen que los Procesos administrativos complejos son otro problema para enfrentar.

C. Ramírez Olivares (2015), presenta en su tesis la siguiente conclusión: "La mayoría de las Pequeñas y Medianas empresas de la Industria Minera de la IV Región de Chile que fueron encuestadas, admite no usar un Sistema de Ayuda a la Decisión. Consideramos que esta carencia de instrumentos de apoyo a la decisión debe cubrirse, en primer lugar, con un análisis de actores participantes en las decisiones sobre propuestas de acciones de mejoras a la problemática de las PYMES de la Industria Minera de la IV Región mediante herramientas de Análisis de Redes Sociales y análisis de influencias a través de ANP”. (Ramírez 2015). Apreciación: No existe coincidencia con las respuestas y apreciaciones de expertos o mineros, pero si advierten la necesidad de Capacitación, es decir si expresan la necesidad de actualizarse, por lo que se debe capacitar a los mineros informales.

D. Calsina Paricahua (2017). En su tesis concluye: "Se analizó la ejecución de resultados de los procedimiento de formalización minera por la vía extraordinaria del Gobierno Regional Puno, determinando las dificultades tales como: la obtención de la acreditación de titularidad, contrato de cesión, acuerdo o contrato de explotación sobre la concesión minera, autorización del terreno superficial, obtención de la licencia o autorización de uso de aguas, por otro lado los sujetos de formalización no pretenden formalizar y la autoridad competente presenta dificultades para atender las demandas del proceso de formalización minera" (Calsina Paricahua, 2017). Apreciación: Se reitera la situación de la titularidad y uso de la superficie, a lo que sumó el problema del agua, aun así se agrega que menciona el estudio, indican que deben hacer menos complejo el Plan de Manejo Ambiental, pero es discrepante con las políticas de Estado, pero además la tesis citada indica la necesidad de atender la problemática ambiental que la minería de cualquier índole afecta al ambiente.

E. Romero Zegarra (2017): precisa en su tesis: "Los mineros del sector de Fortuna señalan que dicha política estuvo orientada a erradicar la minería a pequeña escala, manifestada en un marco normativo adverso a la actividad minera aluvial, en la falta de interés y voluntad política para solucionar problemas en la implementación del proceso de formalización como fue: el escaso presupuesto, ausencia de capacitación y logística y la deficiente articulación y coordinación entre los diferentes sectores involucrados en el proceso de formalización. También podemos sumarle una deficiente comunicación y negociación entre el sector minero y el gobierno, pese a las mesas de trabajo y reuniones convocadas por este. Puesto que no hubo capacidad de escuchar las propuestas y argumentos del sector minero informal, que permita llegar a una solución aceptada por ambas partes. También existe un reconocimiento de todos aquellos actores sociales (gobierno, ONG y gremios) que se encuentran vinculados a la minería informal o que de alguna manera se han visto afectados por las actividades mineras en el sector de Madre de Dios, sobre la importancia económica y social de la actividad minera y de la necesidad de una política de formalización en favor de la sostenibilidad ambiental. Respecto a las deficiencias del proceso de formalización minera reconocen que es necesario realizar una serie de modificaciones al marco normativo que no dio facilidades para la formalización del sector minero informal en Madre de Dios". Apreciación: Indica que la problemática de la formalización es compleja y tiene que ver con muchos aspectos que la presente tesis ha identificado y se corrobora con el estudio que se presentó, pero además de todo, el problema básicamente es la capacitación, la asistencia técnica y profesional y la voluntad política para impulsar estos procesos.

F. Ramírez Olivares (2015): "Referente al proceso de formalización este proceso no promueve ningún cambio solo reprime la actividad minera del pequeño productor minero y del productor minero artesanal". Apreciación: En los últimos tres años, no se aprecia que la Norma o D.L. 1293 contribuya, porque se dificulta su interpretación o su ejecución discrepante y limitada.

Sin duda se puede coincidir que existen problemas básicos para la formalización y que se proponen resolverlos para mejorar el proceso de formalización, resumiendo estos retos son:

- Agilizar la titularidad o autorización de uso de los terrenos superficiales a ser explotados.

- Erradicar la corrupción de las instancias de gobierno.

- Simplificar los procesos de formalización.

- Fortalecer las capacidades de los pequeños mineros y mineros artesanales.

- Facilitar e intermediar el contrato de cesión o contrato de explotación respecto de la concesión minera.

\section{CONCLUSIONES}

- En el estudio original se ha apreciado en los resultados obtenidos que el mayor porcentaje de mineros informales inscritos para el proceso de 
formalización provienen de la región Arequipa, ya que son la $5^{\mathrm{a}}$ parte $(20.03 \%)$ de toda la población total de ellos, siendo la mayoría personas naturales en relación a personas jurídicas, de la región Arequipa se aprecia que el $44.87 \%$ provienen de la provincia de Caravelí, el número de esos mineros son de 6270 mineros al 6 de septiembre del 2020, de los cuales ya se han formalizado 436 mineros, de los cuales 393 son personas naturales y 43 tienen personería jurídica, siendo el caso de mayor formalización en distritos como Chaparra y Huanuhuanu.

- De acuerdo a la encuesta practicada a los expertos e involucrados en formalización de la minería informal se puede apreciar que los conocimientos de estos expertos es bastante bueno de acuerdo a sus referencias y respuestas, quienes indican que el D.L. $\mathrm{N}^{\circ} 1293$ es adecuado e importante para la formalización minera, por lo que se comprende que los procesos de gestión técnicos y ambientales son favorables para la formalización de la minería en pequeña escala en la provincia de Caravelí Arequipa, pues contribuyen a fortalecer sus capacidades integrales y controlar mejor su gestión ambiental, de acuerdo a los resultados que se tiene, sin embargo de ellos existe una dificultad para mejorarlos y es la Acreditación de la titularidad de la concesión minera.

- Se ha podido apreciar que los conocimientos y uso de instrumentos de gestión ambiental son incipientes en los pequeños mineros y mineros artesanales de la provincia de Caravelí - Arequipa, porque de 32 encuestados, 23 indicaron que el proceso de formalización es muy complejo, a pesar de los muchos años dedicados a la minería.

- Las condiciones de desarrollo y explotación de la pequeña minería y minería artesanal en la provincia de Caravelí - Arequipa no son apropiados, existe informalidad y evidencias de daño ambiental.

- Una situación que develan los expertos y los mineros que aseguran de acuerdo a los resultados obtenidos de la existencia de funcionarios corruptos que no permiten el desarrollo del proceso de formalización de manera apropiada, como lo indica la Tabla 18, de 16 expertos, la mitad indica que es el aspecto que no permite el Proceso de formalización, sumado a ello situaciones como los Procesos administrativos complejos y autoridades contemplativas, y los mineros involucrados y encuestados, y en la Tabla 28 precisa que 19 de 32 de ellos reconocen que el problema principal es ese, seguido igualmente de Procesos administrativos complejos y de Autoridades contemplativas.

- Es importante considerar que mineros y expertos también consideran muy importante la capacitación para poder fortalecer el proceso de formalización minera como lo expresan, los expertos en la tabla 19: 5 lo consideran Muy necesario y 1 como muy necesario, y de los mineros informales en la Tabla 29 en la que 24 de ellos lo consideran Necesario y 3 Muy necesario.

\section{REFERENCIAS}

Arias Odón, F. G. (2010). El proyecto de investigacion sexta edicion. In Editoral Episteme (Issue May). Universidad Católica Andrés Bello, UCAB. https://www.researchgate. net/publication/301894369_EL_PROYECTO_DE_ INVESTIGACION_6a_EDICION

Calsina Paricahua, L. G. (2017). Evaluación del proceso de formalización minera en la vía extraordinaria del Gobierno Regional de Puno [Universidad Nacional del Altiplano (Perú)]. http://repositorioslatinoamericanos.uchile.cl/ handle/2250/3275820

Aprueban Reglamento de Organización y Funciones del Ministerio del Ambiente, Diario El Peruano 23 (2008). https://www.minam.gob.pe/wp-content/uploads/2017/09/ DS-007-2017-MINAM.pdf

Díaz Lazo, J. H. (2019). Indicadores ambientales en una minería sostenible. Rev. Del Instituto de Investigación FIGMMGUNMSM, 22(43), 37-44. https://revistasinvestigacion. unmsm.edu.pe/index.php/iigeo/article/view/16684

Díaz Tobar, J. C. (2013). Diseño de una estrategia de intervención basada en el desarrollo de capital humano para la minería artesanal. Tesis.Uchile.Cl, 53(9), 1-139. http://repositorio. uchile.cl/handle/2250/114031

Guzmán, H., Serrano, L. Á., \& Dimate, E. (2019). La minería artesanal en Colombia y su tránsito de lo legal a lo ilegal en el periodo 1978 - 2018 [Universidad Cooperativa de Colombia.]. http://repository.ucc.edu.co:8082/ bitstream/20.500.12494/15468/13/2019 Guzman Serrano_Dimete_minería_artesanal_Colombia_legal_ ilegal_1978-2018. $\overline{\text { pdf }}$

Loaiza, E., Zárate, H., \& Galloso, A. (2008). Mineralización y explotación minera artesanal en la Costa Sur Media del Perú - [Boletín E 4]. In Instituto Geológico, Minero y Metalúrgico - INGEMMET. https://repositorio.ingemmet. gob.pe/handle/20.500.12544/351

Luján, A. (2008). Guía para la Minería Artesanal y de Pequeña Escala Responsable. In Red por la Minería Responsable. http://www.ecosad.org/phocadownloadpap/la rama dorada guia para mineria artesanal y de pequena escala responsable.pdf

Medina Cruz, G. (2014). Formalización de la minería en pequeña escala. Proyecto Better Gold Initiative. https://www.projektconsult.de/newsletter/downloads/631394/formalizacion_ mineria_pequena_escala.pdf

Meza, L. A. J. C. I. (2014). Modelo de desarrollo sostenible en la pequeña minería subterránea: caso Kinacox (ESAN (ed.); Serie Gere, Issue Smal Mining industry in Peru). https://www.esan.edu.pe/publicaciones/serie-gerencia-parael-desarrollo/2014/modelo-de-desarrollo-sostenible-en-lapequena-mineria-subterranea-caso-kinacox/

OEFA. (2016). La vinculación y retroalimentación entre la certificación y la fiscalización ambiental. Organismo de 
Evaluacion y Fiscalizacion Ambiental - OEFA. http://www. oefa.gob.pe/?wpfb_dl=17031

Ramírez Olivares, E. R. (2015). Análisis de la problemática de las PYMES de la industria minera de la IV Región de Chile. Estudio de actores participantes mediante ARS y $A N P$ [Universidad Politécnica de Valencia]. https://dialnet. unirioja.es/servlet $/$ tesis? codigo $=74618$

Romero, A., Garay, E. T., \& Gallarday, T. (2016). TECNOLOGÍA MINERA EN LA EXTRACCIÓN ARTESANAL DEL ORO. Revista Del Instituto de Investigación de La Facultad de Ingeniería Geológica, Minera, Metalurgica y Geográfica, 17(33), 93-97. https://revistasinvestigacion.unmsm.edu.pe/ index.php/iigeo/article/view/11507/10344

Romero Zegarra, K. I. (2017). Proceso de formalización minera: Políticas ambientales y respuestas del sector minero informal a pequeña escala en el poblado Fortuna de Laberinto, Madre de Dios 2012 - 2014 [Pontificia Universidad Católica del Perú]. In Tesis (Issue 48). https://tesis.pucp.edu.pe/ repositorio/handle/20.500.12404/9349
TECSUP. (2020). Operación de Plantas de Procesamiento de Minerales. Programas-Academicos. https://www.tecsup. edu.pe/programas-academicos/carreras-profesionales-3anos/operacion-de-plantas-de-procesamiento-de-minerales

Torres Guerra, J. A., Mejía Cáceres, D., Moreyra Ramos, P., Oré Grados, J., \& Oscco Barrientos, S. (2021). Geometalurgia y el futuro de la minería digital en el Perú. Revista Del Instituto de Investigación de La Facultad de Minas, Metalurgia y Ciencias Geográficas, 24(47), 163-179. https://doi. org/10.15381/iigeo.v24i47.20661 